Supporting Information for

\title{
Metabolic engineering for improved curcumin biosynthesis in
}

\section{Escherichia coli}

Jieyuan $\mathrm{Wu}^{\S, \dagger}$, Wei Chen ${ }^{\S}$, Yutong Zhang ${ }^{\ddagger}$, Xuanxuan Zhang ${ }^{\S, \dagger}$, Jian-Ming Jin ${ }^{*, \neq}$,

Shuang-Yan Tang ${ }^{*} \S$

${ }^{\S}$ CAS Key Laboratory of Microbial Physiological and Metabolic Engineering, State

Key Laboratory of Microbial Resources, Institute of Microbiology, Chinese Academy

of Sciences, Beijing, China.

${ }^{\ddagger}$ Beijing Key Laboratory of Plant Resources Research and Development, Beijing

Technology and Business University, Beijing, China.

${ }^{\dagger}$ University of Chinese Academy of Sciences, Beijing, China.

* Corresponding author.

Shuang-Yan Tang: Institute of Microbiology, Chinese Academy of Sciences, No. 1

West Beichen Road, Chaoyang District, Beijing 100101, China

Tel: 86-10-64806140;

E-mail: tangsy@im.ac.cn

Jian-Ming Jin: Beijing Technology and Business University, No. 11 Fucheng Road,

Haidian District, Beijing 100048, China

Tel: 86-10-68984917;

E-mail: jinjianming@btbu.edu.cn 
Table of Contents

\begin{tabular}{|c|c|c|}
\hline Section & Contents & Page \\
\hline \multirow{3}{*}{ Tables } & S1. Strains and plasmids used in this study. & S3 \\
\cline { 2 - 3 } & S2. Primers used in this study. & S5 \\
\cline { 2 - 3 } & S3. Optimized gene sequence encoding CUS. & S6 \\
\hline \multirow{2}{*}{ Figures } & $\begin{array}{c}\text { S1. Curcumin synthesized by the biosynthetic pathway was } \\
\text { detected by HPLC. }\end{array}$ & S7 \\
\cline { 2 - 3 } & $\begin{array}{c}\text { S2. Sequence and minimum free energy of the 5' region of } \\
\text { CUS wild-type and mutant M1. }\end{array}$ & S8 \\
\hline References & References for Supporting Information. & S9 \\
\hline
\end{tabular}


Table S1. Strains and plasmids used in this study.

\begin{tabular}{|c|c|c|}
\hline Strains and plasmids & Relative characteristics & Resources \\
\hline \multicolumn{3}{|l|}{ Strains } \\
\hline BW25113 & $\begin{array}{l}\text { lacl }{ }^{\mathrm{q}}, r r n B 3, \Delta l a c Z 4787, h s d R 514, \\
\Delta(\operatorname{araBAD}) 567, \Delta(r h a B A D) 568, r p h-1\end{array}$ & Lab collection \\
\hline MC1061 & $\begin{array}{l}\text { hsdR, mcrB, araD139, } \Delta(\text { araABC-leu }) 7679, \\
\Delta l a c X 74, \text { galU, galK, rpsL, thi }\end{array}$ & Lab collection \\
\hline BL21 (DE3) & $\begin{array}{l}\text { ompT, hsd } S_{\mathrm{B}}\left(r_{B}^{-} m_{B}^{-}\right), \text {gal }(\lambda c 1857, \text { ind } 1, \\
\text { Sam7,nin5, lacUV5-T7 gene } 1), d c m(\mathrm{DE} 3)\end{array}$ & Lab collection \\
\hline E. coli $\triangle$ poxB ::acs $\triangle a d h E$ & $\begin{array}{l}\text { BW } 25113 \text { with deletion of poxB, } a d h E \text { and } \\
\text { overexpression of acs }\end{array}$ & (1) \\
\hline CUR01 & $\begin{array}{l}\text { BW25113 with deletion of poxB, } a d h E \text { and } \\
f a b F \text { and overexpression of } a c s\end{array}$ & This study \\
\hline CUR02 & CUR01 with deletion of curA & This study \\
\hline \multicolumn{3}{|l|}{ Plasmids } \\
\hline pBAD18-Kan & pMB 1 origin, $\operatorname{ara\mathrm {BAD}}$ promoter, $\mathrm{Km}^{\mathrm{R}}$ & Biovector, NTCC \\
\hline pCOLADuet-1 & ColA origin, $T 7$ lac promoter, $\mathrm{Km}^{\mathrm{R}}$ & Biovector, NTCC \\
\hline pYB1a & mutant $\mathrm{p} 15 \mathrm{~A}$ origin, $\operatorname{ara} \mathrm{BAD}$ promoter, $\mathrm{Ap}^{\mathrm{R}}$ & (1) \\
\hline pLB1k & $\begin{array}{l}\text { R6K origin, } \lambda \text { pir protein, araBAD promoter, } \\
\mathrm{Km}^{\mathrm{R}}\end{array}$ & (2) \\
\hline pAk & ColA origin, araBAD promoter, $\mathrm{Km}^{\mathrm{R}}$ & This study \\
\hline pYk & mutant $\mathrm{p} 15 \mathrm{~A}$ origin, araBAD promoter, $\mathrm{Km}^{\mathrm{R}}$ & This study \\
\hline $\mathrm{pLk}$ & $\mathrm{R} 6 \mathrm{~K}$ origin, $\operatorname{ara\mathrm {BAD}}$ promoter, $\mathrm{Km}^{\mathrm{R}}$ & This study \\
\hline pACA (pAk-CUS-4AT) & $\begin{array}{l}\text { ColA origin, } \operatorname{araBAD} \text { promoter controlled } \\
\text { CUS and } 4 \mathrm{AT}, \mathrm{Km}^{\mathrm{R}}\end{array}$ & This study \\
\hline pYCA (pYk-CUS-4AT) & $\begin{array}{l}\text { Mutant p15A origin, araBAD promoter } \\
\text { controlled CUS and } 4 \mathrm{AT}, \mathrm{Km}^{\mathrm{R}}\end{array}$ & This study \\
\hline pLCA (pLk-CUS-4AT) & $\begin{array}{l}\text { R6K origin, } \operatorname{araBAD} \text { promoter controlled } \\
\text { CUS and } 4 \mathrm{AT}, \mathrm{Km}^{\mathrm{R}}\end{array}$ & This study \\
\hline pYAC (pYk-4AT-CUS) & $\begin{array}{l}\text { Mutant p15A origin, } \operatorname{araBAD} \text { promoter } \\
\text { controlled } 4 \mathrm{AT} \text { and CUS, } \mathrm{Km}^{\mathrm{R}}\end{array}$ & This study \\
\hline pYk-CUS M1-4AT & $\begin{array}{l}\text { Mutant p15A origin, araBAD promoter } \\
\text { controlled CUS M1 and } 4 \mathrm{AT}, \mathrm{Km}^{\mathrm{R}}\end{array}$ & This study \\
\hline pET28a & pBR322 origin, $\mathrm{T} 7$ promoter, $\mathrm{Km}^{\mathrm{R}}$ & Biovector, NTCC \\
\hline pET28a-CUS & $\begin{array}{l}\text { pBR322 origin, } T 7 \text { promoter controlled CUS, } \\
\mathrm{Km}^{\mathrm{R}}\end{array}$ & This study \\
\hline
\end{tabular}




\begin{tabular}{|c|c|c|}
\hline pET28a-CUS M1 & $\begin{array}{l}\text { pBR322 origin, } \mathrm{T} 7 \text { promoter controlled CUS } \\
\mathrm{M} 1, \mathrm{Km}^{\mathrm{R}}\end{array}$ & This study \\
\hline pTrc99a & pBR322 origin, $P_{\text {trc }}$ promoter, $A p^{R}$ & Lab collectior \\
\hline pTrc99a-Almgs & pBR322 origin with $\mathrm{P}_{\text {trc }}$ controlled almgs, $\mathrm{Ap}^{\mathrm{R}}$ & This study \\
\hline pKD13 & $\mathrm{R} 6 \mathrm{~K} \gamma$ origin, kan cassette template, $\mathrm{Ap}^{\mathrm{R}}, \mathrm{Km}^{\mathrm{R}}$ & (3) \\
\hline pCP20 & $\begin{array}{l}\text { pSC101 origin, FLP recombinase expression, } \\
\mathrm{Ap}^{R}, \mathrm{Cm}^{\mathrm{R}}\end{array}$ & (4) \\
\hline pKD46 & $\begin{array}{l}\mathrm{R} 101 \text { origin, } \operatorname{ara\mathrm {BAD}} \text { promoter controlled } \\
\text { Red recombinase, } \mathrm{Ap}^{\mathrm{R}}\end{array}$ & (3) \\
\hline
\end{tabular}


Table S2. Primers used in this study.

\begin{tabular}{|c|c|}
\hline Primers & Sequences $\left(5^{\prime}-3^{\prime}\right)$ \\
\hline CurA up-For & TCATCAATCAATCGGCTTAACAATTTAC \\
\hline CurA up-Rev & GAAGCAGCTCCAGCCTACACAATGCTCTCCTGTGCTTTTGGC \\
\hline CurA down-For & GGTCGACGGATCCCCGGAATAGTTTCAACGGCGGCGTAAG \\
\hline CurA down-Rev & AACAGCCAGAGCAACTGCC \\
\hline P1 & GTGTAGGCTGGAGCTGCTTC \\
\hline P4 & ATTCCGGGGATCCGTCGACC \\
\hline pBAD18-Kan-For & AACGCCAGCAACGCGGCCTTT \\
\hline pBAD18-Kan-Rev & TAAATCAATCTAAAGTATATATGAGTAAACTTGGTCTG \\
\hline ColA-For & ATATACTTTAGATTGATTTAGCTAGCGCAGAAACGTCCTAG \\
\hline ColA-Rev & AAGGCCGCGTTGCTGGCGTTAGTGATGGTGTCGGGAATC \\
\hline p15A-For & ATATACTTTAGATTGATTTAGTGCGTCAGCAGAATATGTGATAC \\
\hline p15A-Rev & $\begin{array}{l}\text { AAGGCCGCGTTGCTGGCGTTGTGCTACATTTGAAGAGATAAA } \\
\text { TTGCACTG }\end{array}$ \\
\hline R6K-For & ATATACTTTAGATTGATTTACCATGTCAGCCGTTAAGTGTTC \\
\hline R6K-Rev & AAGGCCGCGTTGCTGGCGTTTCACCCCTTAGCTTTTTTGG \\
\hline pYk-For & GAATTCGGTGAGCTCGGTCT \\
\hline pYk-Rev & AAGCTTAATTCCTCCTGTTAGCCC \\
\hline C4-CUS-For & TAACAGGAGGAATTAAGCTTATGGCGCCGACCACCACC \\
\hline C4-CUS-Rev & TTAATTCACATGGCTGGT \\
\hline C4-4AT-For & $\begin{array}{l}\text { ACCAGCCATGTGAATTAAAAGGAGATATACATGGCGCCACAA } \\
\text { GAACAAGC }\end{array}$ \\
\hline C4-4AT-Rev & $\begin{array}{l}\text { AGACCGAGCTCACCGAATTCTCACAATCCATTTGCTAGTTTTG } \\
\text { CCCTC }\end{array}$ \\
\hline 4C-4AT-For & TAACAGGAGGAATTAAGCTTATGGCGCCACAAGAACAAGC \\
\hline 4C-4AT-Rev & TCACAATCCATTTGCTAGTTTTGCCCTC \\
\hline 4C-CUS-For & $\begin{array}{l}\text { AACTAGCAAATGGATTGTGAAAGGAGATATACATGGCGCCGA } \\
\text { CCACCACC }\end{array}$ \\
\hline 4C-CUS-Rev & AGACCGAGCTCACCGAATTCTTAATTCACATGGCTGGT \\
\hline CUS-For & ATGGCGCCGACCACCACC \\
\hline CUS-Rev & TTAATTCACATGGCTGGT \\
\hline CUS-NdeI For & GGGGGGGATCCATATGGCGCCGACCACCACC $^{\mathrm{a}}$ \\
\hline CUS-XhoI Rev & CGGCTCGAGTTAATTCACATGGCTGGT \\
\hline pTrc99a-Gibson-For & GGCTGTTTTGGCGGATGAGAG \\
\hline pTrc99a-Gibson-Rev & GGTCTGTTTCCTGTGTGAAATTGTTATC \\
\hline Almgs-Gibson-For & $\begin{array}{l}\text { TTTCACACAGGAAACAGACCATGCGTATTGGTATCTTCAGCG } \\
\text { AGGC }\end{array}$ \\
\hline Almgs-Gibson-Rev & $\begin{array}{l}\text { TCTCATCCGCCAAAACAGCCTTATTTCTTGTTCAGTTTCTTGT } \\
\text { TGTTTTTATCG }\end{array}$ \\
\hline
\end{tabular}

[a] Restriction sites were underlined. 
Table S3. Optimized gene sequence encoding CUS.

\begin{tabular}{l|l}
\hline Gene & Sequence \\
\hline CUS & ATGGCGCCGACCACCACCATGGGCAGCGCGCTGTATCCGCTGGGCGAAAT \\
GCGTCGTAGCCAGCGCGCGGATGGCCTGGCAGCAGTGCTGGCAATTGGCA \\
CCGCCAACCCGCCGAACTGCGTGACCCAGGAAGAAATTCCGGATTTTTAC \\
TTTCGCGTGACCAACAGCGATCATCTGACCGCGCTGAAAGATAAATCAAA \\
CGCATTTGCCAGGAAATGGGCGTGCAGCGCCGCTATCTGCACCACACCGA \\
AGAAATGCTGAGCGCCCATCCGGAATTTGTGGATCGTGATGCCCCGAGCCT \\
GGATGCGCGTCTGGATATTGCCGCGGATGCCGTGCCGGAACTGGCCGCAG \\
AAGCCGCAAAAAAGGCGATTGCGGAATGGGGCCGCCCGGCGGCAGATATT \\
ACCCACCTGGTGGTGACCACCAATAGCGGCGCGCACGTGCCGGGCGTGGA \\
TTTTCGTCTGGTGCCGCTGCTGGGCCTGCGTCCGTCAGTGCGTCGTACCAT \\
GCTGCACCTGAACGGCTGCTTTGCGGGCTGCGCCGCGCTGCGTCTGGCAA \\
AAGATCTGGCGGAAAACAGCCGCGGCGCCCGCGTTCTGGTGGTGGCAGCA \\
GAACTGACCCTGATGTATTTTACCGGCCCGGATGAAGGCTGCTTTCGCACC \\
CTGCTGGTGCAGGGCCTGTTTGGCGATGGCGCCGCGGCAGTGATTGTGGG \\
CGCCGATGCGGATGATGTGGAACGTCCGCTGTTTGAAATTGTGAGCGCCGC \\
CCAGACCATTATTCCGGAAAGCGATCATGCGCTGAACATGCGCTTTACCGA \\
ACGCCGTCTGGATGGCGTGCTGGGCCGTCAGGTGCCGGGTCTGATTGGCG \\
ATAATGTGGAACGCTGCCTGCTGGATATGTTTGGCCCGCTGCTGGGTGGCG \\
ATGGCGGCGGTGGTTGGAACGATCTGTTTTGGGCCGTGCATCCGGGCAGC \\
AGCACCATTATGGATCAGGTGGATGCGGCCCTGGGCCTGGAACCGGGTAA \\
ACTGGCGGCGAGCCGTCGTGTGCTGAGCGATTACGGCAACATGAGCGGCG \\
CGACCGTGATTTTTGCGCTGGATGAACTGCGTCGCCAGCGCAAAGAAGCG \\
GCCGCGGCAGGTGAATGGCCGGAACTGGGCGTGATGATGGCGTTTGGCCC \\
GGGCATGACCGTGGATGCCATGCTGCTGCATGCCACCAGCCATGTGAATTA \\
A
\end{tabular}



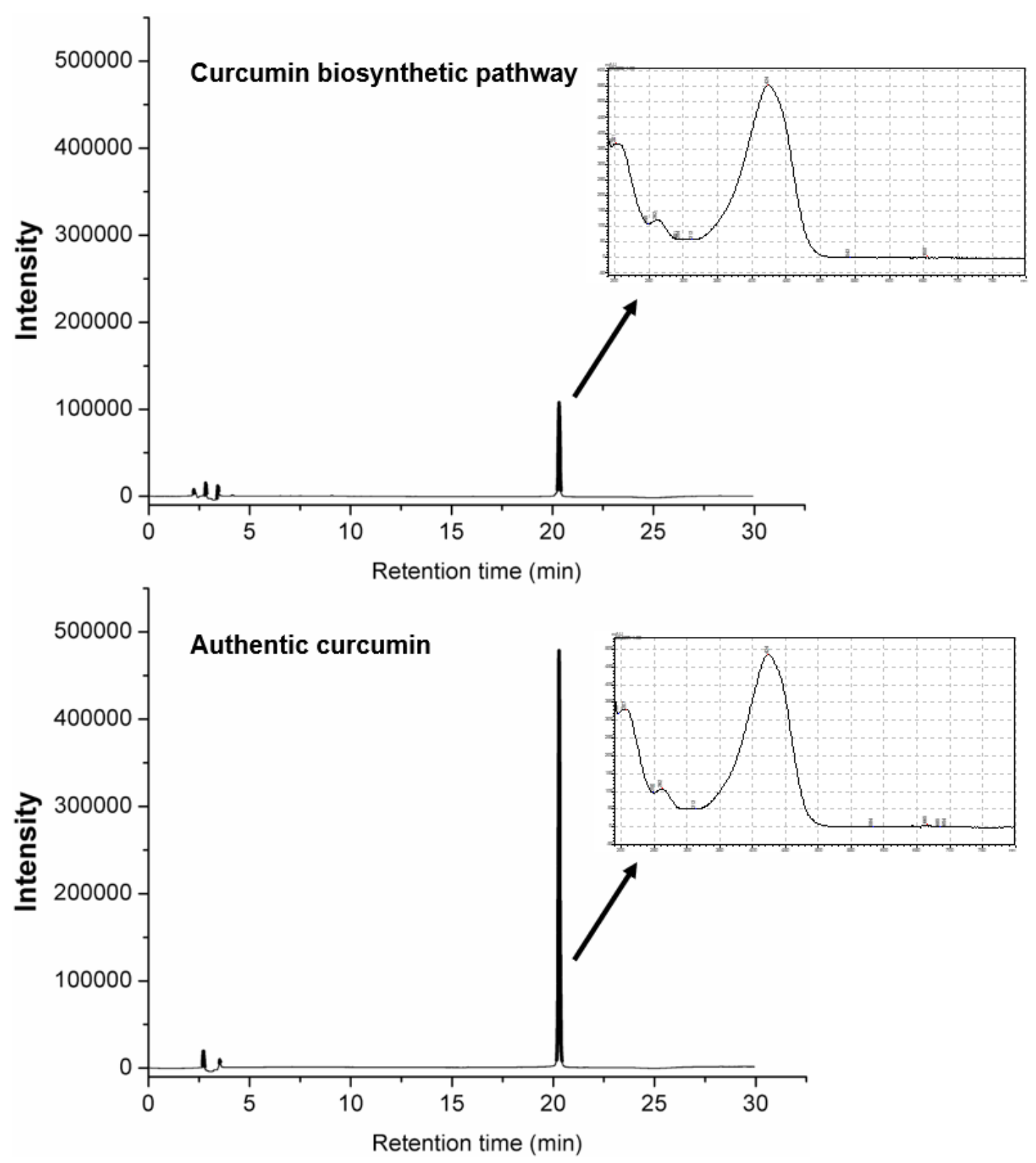

Figure S1. Curcumin synthesized by the biosynthetic pathway was detected by HPLC. Strain BW25113 harboring plasmid pYCA was cultured for $12 \mathrm{~h}$. 
atggcgccgaccaccaccatgggcagcgcgctgtatccgc MFE structure at $30.0 \mathrm{C}$

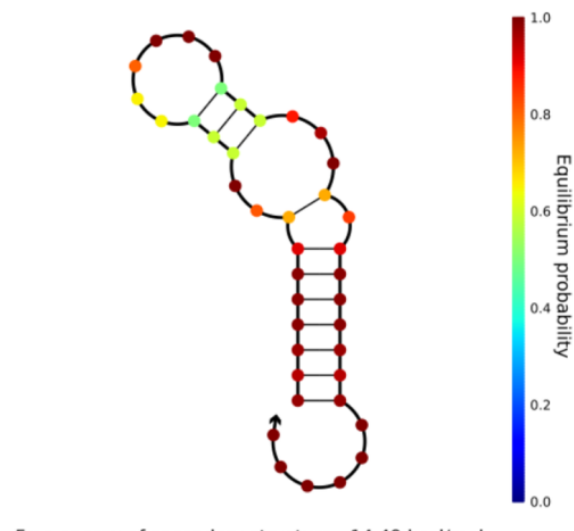

CUS-WT atggcgccgaccaccaccatgggcagcacgctgtatccgc MFE structure at $30.0 \mathrm{C}$

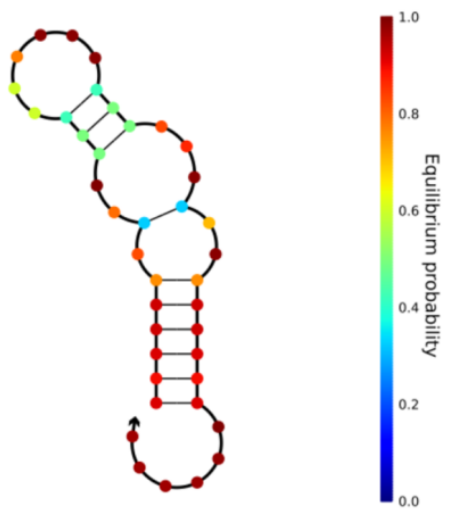

Free energy of secondary structure: $-9.44 \mathrm{kcal} / \mathrm{mol}$ CUS-M1 and M8

Figure S2. Sequence and minimum free energy of the 5' region of mRNA of CUS wild-type and mutant $\mathrm{M} 1$ and M8, calculated at $30^{\circ} \mathrm{C}$. 


\section{References}

(1) Cui, Q.; Zhou, F.; Liu, W.; Tao, Y., Avermectin Biosynthesis: Stable Functional Expression of Branched Chain Alpha-keto Acid Dehydrogenase Complex From Streptomyces avermitilis in Escherichia coli by Selectively Regulating Individual Subunit Gene Expression. Biotechnol. Lett. 2017, 39 (10), 1567-1574.

(2) Liu, B.; Xiang, S.; Zhao, G.; Wang, B.; Ma, Y.; Liu, W.; Tao, Y., Efficient Production of 3-hydroxypropionate From Fatty Acids Feedstock in Escherichia coli. Metab. Eng. 2019, 51, 121-130.

(3) Datsenko, K. A.; Wanner, B. L., One-step Inactivation of Chromosomal Genes in Escherichia coli K-12 Using PCR Products. Proc. Natl. Acad. Sci. U. S. A. 2000, 97 (12), 6640-6645.

(4) Cherepanov, P. P.; Wackernagel, W., Gene Disruption in Escherichia coli: TcR and KmR Cassettes with the Option of Flp-catalyzed Excision of the Antibiotic-resistance Determinant. Gene 1995, 158 (1), 9-14. 